\title{
Mord und Totschlag
}

\author{
Hans-Ludwig Kröber
}

Online publiziert: 29. September 2015

(C) Springer-Verlag Berlin Heidelberg 2015

\section{Murder and homicide}

Der Bundesjustizminister Heiko Maas hat 2014 eine vor mehr als dreißig Jahren eingeschlafene Diskussion über eine Reform der strafrechtlichen Normierung der Tötungsdelikte aufgegriffen, befördert durch Länderinitiativen und Vorschläge des Deutschen Anwaltvereins (DAV) sowie von weiteren Juristen. Der DAV hat seinen Vorschlag am 29.04.2014 in Anwesenheit des Ministers vorgestellt; er beinhaltet eine Abschaffung der Begriffe Mord und Totschlag und einen einheitlichen Tötungstatbestand, der mit zeitiger oder lebenslanger Freiheitsstrafe zu ahnden sei. Wie schuldangemessen zwischen der Mindeststrafe von 8 Jahren und lebenslang zu differenzieren ist, wird nicht ausgeführt. Betont wurde in der Diskussion, dies werde - in Anlehnung an die bisherige Rechtsprechung (die anhand der angeblich nazistischen Mordmerkmale erfolgte) - den Richtern schon gelingen. In der Diskussion stand Albin Eser auf, der nach wie vor jugendfrische Verfasser des Reformvorschlags für den Deutschen Juristentag 1980, und wies darauf hin, dass just diese Weglassung jeder weiteren Mordqualifikation im Strafgesetzbuch der erste Vorschlag des Staatssekretärs Roland Freisler gewesen sei. Auf Kritik an der mangelnden Bestimmtheit habe er geantwortet, der deutsche Richter werde schon fraglos erkennen, wenn er einen Mörder vor sich hat. Die Bedenken der Fachleute aus dem Justizministerium hätten sich dann aber durchgesetzt, sodass es - in

Prof. Dr. H.-L. Kröber ( $\bowtie)$

Institut für Forensische Psychiatrie,

Charité - Universitätsmedizin Berlin,

Oranienburger Str. 285,

13437 Berlin, Deutschland

E-Mail: mail@hlkroeber.de
Anlehnung an die Schweizer, wesentlich durch Carl Stooss beeinflusste Gesetzgebungsdiskussion - zu der Formulierung der heute noch gültigen Mordmerkmale kam.

Der ursprüngliche Vorschlag Freislers wäre wohl der für eine willkürliche Rechtsanwendung gefährlichere Vorschlag gewesen: Es genügte das Erkennen der Täterpersönlichkeit als „Mörder“. Der „Mörder“ wird mit dem Tode bestraft, „Mörder“ und des Todes war man auch, wenn es beim Mordversuch geblieben war.

Geändert hat sich seit 1941, dass in der Bundesrepublik mit Verabschiedung des Grundgesetzes 1949 die Todesstrafe abgeschafft wurde (in der DDR im Juli 1987), und dass die Mordmerkmale durch die Rechtsprechung von Bundesverfassungsgericht und Bundesgerichtshof eine zweifelsfrei rechtsstaatliche und grundgesetzkonforme Auslegung erhielten. Geblieben war aus der NS-Zeit die Rede vom „Mörder“ und vom „Totschläger“ statt wie sonst im StGB von Taten (also von Mord oder Totschlag).

Die gesetzliche Fassung der Normen über die Tötungsdelikte zu überdenken - bisher im 70-Jahres-Rhythmus erscheint aber auch dann sinnvoll, wenn man den gültigen Normen keinen faschistischen Gehalt zusprechen kann. Diese Diskussion wurde und wird geführt in den Medien, den Fachzeitschriften und in der „Kommission zur Reform der Tötungsdelikte" beim Bundesministerium der Justiz und für Verbraucherschutz (BMJV), die von Mai 2014 bis Mai 2015 zahlreiche Fachleute als Referenten gehört hat, und am 12. Juni 2015 auch auf der 19. Berliner Junitagung für Forensische Psychiatrie und Psychologie, die noch stärker den Schwerpunkt auf unser (begrenztes) empirisches Wissen über Tötungsdelinquenten gelegt hat. Aus beiden Quellen ist das vorliegende Schwerpunktheft von Forensische Psychiatrie, Psychologie, Kriminologie gespeist.

Es beginnt mit der Übersicht von Jörg Kinzig über die Häufigkeit der Tötungsdelikte im Verlauf der letzten Jahr- 
zehnte, mit dem in der Öffentlichkeit kaum wahrgenommenen Befund eines drastischen Rückgangs der versuchten und vollendeten Tötungsdelikte in den letzten 15 Jahren um ein Viertel. Andererseits scheinen sich die Verbüßungsdauern verlängert zu haben (in ähnlichem Umfang im gesamten Straf- und Maßregelvollzug), sodass es bis $2011 \mathrm{zu}$ einem stetigen Anstieg der Insassen mit lebenslanger Strafe auf über 2000 Personen kam (bei 58.000 Strafgefangenen insgesamt). Zudem stieg die Quote der wegen Mordes Verurteilten, die keine verminderte Schuldfähigkeit und mithin „lebenslang“" erhielten, von 49\% 1990, 55\% 1999 auf Werte, die seit 2001 um 70\% schwanken (2013: 74\%). Kinzig sieht dies als „härtere Gangart der Justiz“, wichtigste Ursache dürfte aber sein, dass durch Änderung der BGH-Positionen nicht mehr jede unbelegte Einrede von Berauschung bei der Tat zur Zuerkennung verminderter Schuldfähigkeit führt.

Es folgen zwei Beiträge der Junitagung zu bestimmten Tätergruppen. Norbert Leygraf beleuchtet den vermeintlich wohlbekannten „Beziehungstäter" und verdeutlicht, welch weites Spektrum von Tatmotiven und Tatkonstellationen hier anzutreffen ist; der unglückliche, verlassene Romantiker, der sich zu einer Affekttat hinreißen lässt, ist die Ausnahme. Eher schon waren psychisch kranke Täter zu identifizieren.

Wolfgang Würz, zuletzt Leiter der Abteilung islamistischer Terrorismus beim Bundeskriminalamt, verdeutlicht aus Sicht des Kriminalbeamten die Schwierigkeiten, bei der präventiven Gefahrenabwehr, also der Verhinderung terroristischer Anschläge, einen bestimmten Risikotyp auszumachen, nach dem eine Rasterfahndung erfolgen könnte. Gestützt auf die persönliche Kenntnis solcher Täter verdeutlicht er, dass zum Töten entschlossene junge Männer keineswegs eine markante psychische Pathologie oder überschießende Gewaltbereitschaft haben müssen, sondern dass die islamistischen Organisationen offenbar Bedürfnisse ansprechen, in eine feste Gemeinschaft zu gehören, etwas Besonderes zu sein und zu leisten, zu kämpfen und eigene Schwächen und Ängste zu überwinden.

Der Beitrag von Dieter Dölling befasst sich mit Rechtstatsachen, also der Frage, wie die Bestimmungen des Strafrechts zu Mord und Totschlag richterlich angewendet werden. Dies betrifft die Fragen, wie häufig die unterschiedlichen Mordmerkmale festgestellt werden und welche Mordmotive in empirischen Untersuchungen gefunden wurden. Speziell befasst er sich mit den Entscheidungen, die für oder gegen die Anwendung der Mordmerkmale „Heimtücke“ und „niedrige Beweggründe“ argumentiert haben und mit den Motiven bei solchen Taten. Dargestellt wird schließlich die Rechtsprechung bei Kindstötungen.

Alexander Ignor vermittelt in seinem Beitrag über die aktuelle Reformdiskussion einen auch dem Nichtjuristen gut verständlichen Überblick über wesentliche Diskussionen in der Reform-Kommission. Wie soll man sich juris- tisch nähern, allein über den schieren Tatbestand der Tötung eines Menschen, ähnlich der Lösung in Österreich, mit dann allein richterlicher Beurteilung, wann die Höchststrafe angemessen ist? Dies war der Vorschlag des DAV. Oder über das Konzept von Regelbeispielen, ,ein besonders schwerer Fall liegt in der Regel dann vor, wenn." Dieses Konzept hat aber eine gewisse Unverbindlichkeit und kann in richterlicher „Gesamtwürdigung“ des Falles leicht umgangen werden. Oder weiter das bestehende "Qualifizierungskonzept", dass bei Vorliegen bestimmter, abschließend benannter Merkmale die Höchststrafe zwingend vorgeschrieben ist? Ignor stellt die Vor- und Nachteile der Modelle wie auch die Diskussion in der Kommission abgewogen dar, auch wenn er schließlich für einen eigenen Vorschlag plädiert, der keine Mehrheit fand. Einigkeit bestand, dass die Ausschließlichkeit der lebenslangen Strafe beim Erfüllen eines Mordmerkmals, also die absolute Strafandrohung, durch eine Regelung für solche Fälle aufgebrochen werden muss, in denen dies wegen der besonderen Lage des Täters oder der Täterin („,Tyrannenmord“) eine grobe Ungerechtigkeit ergeben würde. Speziell befasst sich Ignor mit dem Mordmerkmal der Heimtücke und verdeutlicht die Widersprüche, die sich inzwischen auch im juristischen Verständnis des Begriffs ergeben haben. Auch hier versprechen die Vorschläge der Kommission eine Verbesserung.

Hartmut Schneider, der sich in der Kommission mit Alexander Ignor manch spannende, beiderseits stets respektvolle Diskussion geliefert hat, verdeutlicht als StaatsAnwalt den rechtspolitischen Kontext der Diskussion wie auch der Bestimmungen über die Straftaten gegen das Leben und plädiert in epigrammatischer Kürze für vorsichtige Änderungen, die das Gewaltmonopol des Staates und seinen entsprechenden Anspruch gegenüber dem Bürger nicht beschädigen.

Die Schwerpunktdiskussion über Mord und Totschlag endet in diesem Heft zunächst mit einem Beitrag von HansLudwig Kröber über die Mordmerkmale aus psychiatrischer Sicht, also die Übertragbarkeit von Rechtsbegriffen in eine psychologische Sichtweise. Unterschieden wird zwischen den Merkmalen, die auf eine bestimmte, besonders verwerfliche Motivation abheben (Mordlust, Geschlechtsgier, Habgier, niedrige Beweggründe), und andererseits tatbezogenen Merkmalen wie heimtückisch, grausam oder mit gemeingefährlichen Mitteln und schließlich Merkmalen, die Funktionen der Tat benennen, nämlich die Ermöglichung oder Verdeckung einer anderen Straftat. Wo es um Motivationen, Gesinnungen und potenzielle Gefährlichkeit geht, ist natürlich der psychiatrische Gutachter involviert, auch wenn er sich nur zur Frage der Schuldfähigkeit äußern soll: Geht es doch um die Frage, wie die Tat zu deuten ist und was dies - im Moment der Tat - über den Täter aussagt. Letztlich wird sich die Strafjustiz weiter mit dem Phantasma des „Mörders“, der vermeintlichen oder tatsächlichen Täterper- 
sönlichkeit auseinandersetzen müssen; je reflektierter und realitätsorientierter, desto besser.

Als Nachzügler zum Schwerpunktthema des letzten Heftes - DSM-5 und die Forensische Psychiatrie - erscheint jetzt der Beitrag von Paul Hoff zum Spannungsverhältnis von Psychopathologie und operationalisierter Diagnostik in der forensischen Psychiatrie. Er beleuchtet den historischen und den konzeptuellen Hintergrund der operationalisierten Diagnostik und die mindestens bis Kraepelin zurückreichende Forderung, eine möglichst deutungsfreie, theoriearme, rein deskriptive Darstellung der psychopathologischen Phänomene zu liefern. Zugleich aber bestand, von Jaspers unterstrichen, die Problematik, dass es in der Wahrnehmung von Fremdpsychischem eben keine fotografische (oder computertomographische) Abbildung geben kann, sondern dass der subjektive psychische Raum eines Anderen nur interaktiv, einfühlend, verstehend erschlossen werden kann. Hoff geht den einzelnen Vorgaben der operationalisierten Diagnostik nach, wie weit sie objektiver, zuverlässiger, theoriearm und in ihrer Mehrdimensionalität reicher auftreten, und erörtert dann die besonderen Prob- leme einer durch Algorithmen sozusagen automatisierten, dem psychiatrischen Sachverständigen nahezu entwundenen Diagnostik im forensischen Feld.

Es folgen die kriminologischen und psychiatrischen Beiträge des Journal-Club. Julia Sieß referiert Befunde zu den Veränderungen kriminalprognostischer Risikofaktoren unter forensisch-psychiatrischer Behandlung. Angelika Treibel berichtet über Studien, die sich mit den Qualitätsund Erfolgskriterien kriminalpolizeilicher Ermittlungsarbeit, speziell die Problematik der Aufklärungsquoten, befassen. Am Ende steht dann vor dem Kongresskalender ein Blitzlicht, das sich diesmal ausnahmsweise auch mit dem Schwerpunktthema befasst und für die Beibehaltung der Androhung der lebenslangen Freiheitsstrafe plädiert. Verbunden mit einer Strafvollstreckung, die dann doch keine endgültige Verstoßung darstellt.

Hans-Ludwig Kröber

Interessenkonflikt H.-L. Kröber gibt an, dass kein Interessenkonflikt besteht. 